\title{
Les diplômés de l'immersion: implications dans le domaine de l'enseignement du français 1
}

\author{
MARJORIE BINGHAM WESCHE*
}

\section{RÉSUMÉ}

Cet article présente les résultats de trois études portant sur les diplômés d'immersion de la région d'Ottawa. La première étude vise à présenter les cours de français offerts à des apprenants de langue seconde de niveau universitaire. Cette étude a été menée dans quatre universités où étudient la majorité des diplômés d'immersion de la région d'Ottawa; soit les universités d'Ottawa, Carleton, Queen's et McGill. L'étude offre une description des choix de cours, des programmes et des services offerts en français depuis 1985. La seconde étude comprenait une enquête sur les études antérieures en français de tous les étudiants anglophones de première année de l'Université d'Ottawa en 1987. De plus, elle cherchait à connaître leurs intentions quant à l'utilisation de leur langue seconde. L'étude a démontré que plus de $20 \%$ de ces étudiants avaient suivi un programme d'immersion et que la majorité voulait poursuivre des études de français. La troisième étude, de type longitudinal, a permis de suivre, de 1985 à 1988, un groupe d'étudiants universitaires diplômés d'immersion. Au début de leurs études universitaires, ces étudiants avaient une compétence fonctionnelle élevée en français (bien que moins élevée que celle d'un locuteur natif). De plus, cette compétence restait élevée tout au long de leurs études universitaires. Cependant, en première comme en troisième année, ils disaient utiliser assez peu leur langue seconde à l'extérieur de la classe et ne suivre que quelques cours en français. Les participants à cette étude ont affirmé qu'ils évitent de suivre leurs cours en français de peur d'obtenir des notes peu élevées ou à cause du nombre restreint de cours optionnels au sein de leur programme universitaire. En s'inspirant de ces raisons évoquées par les étudiants, l'auteure propose des changements aux niveaux pédagogique et administratif afin d'encourager ces étudiants à suivre plus de cours en français.

*Second Language Institute, University of Ottawa. 


\begin{abstract}
This paper reports the results of three studies relating to French immersion graduates in the Ottawa area. The first study concerns post-secondary offerings in French for second language speakers. Conducted at four universities which receive the largest number of these students, Ottawa, Carleton, Queen's and McGill, it describes a variety of courses, programs and services in French available as early as 1985. The second study surveyed all first-year anglophone students at the University of Ottawa in 1987 on their background and future intentions regarding French language study. It revealed that over $20 \%$ had studied in French immersion programs and that there was considerable interest in further French study. The third study was a longitudinal follow-up of immersion graduates in the four universities between 1985 and 1988. It showed that upon beginning university studies, they had very high functional abilities in French (although not native-like), and that these skills were maintained during their university studies. However, in first as well as third year, they reported using French very little outside class and taking only a few classes in it. Starting from the idea expressed by students in both studies that they avoid courses in French for fear of getting lower grades or because they have very restricted electives in their academic programs, the author suggests changes in terms of course offerings and at the administrative level to encourage these students to take more courses in French.
\end{abstract}

\title{
INTRODUCTION
}

Près de 240,000 enfants anglophones sont actuellement inscrits aux programmes d'immersion française au Canada. En Ontario, où ces programmes sont le mieux établis, ceci représente quelque $7 \%$ de la population scolaire. A ce sujet Ottawa est probablement le centre mondial du phénomène de l'immersion. Plus de la moitié des enfants anglophones de la région de la capitale nationale commencent maintenant le jardin d'enfants en français. Evidemment, bon nombre de ces élèves reviennent au programme anglais durant leurs années scolaires, mais un nombre de plus en plus important d'étudiants complètent leurs études dans des programmes bilingues au secondaire. On assiste donc présentement à l'arrivée d'un nouveau type d'étudiant dans les universités, à savoir le diplômé anglophone fonctionnellement bilingue. En 1988, il y a eu plus de 600 diplômés des programmes bilingues au niveau secondaire dans la région d'Ottawa, en plus des centaines d'autres qui ont fait une partie de leurs études dans des classes d'immersion française.

Que savons-nous de ces étudiants sortant de l'immersion? Sont-ils différents dans leurs attitudes, leurs ambitions et leurs habitudes des étudiants qui n'ont pas étudié en immersion? Que veulent-ils faire après leurs études secondaires? Iront-ils à l'université? Si oui, que voudront-ils étudier? Ces étudiants se servent-ils du français hors de la salle de classe? Quelles sont leurs attitudes à 
l'égard des études post-secondaires en français? Qu'attendent-ils des universités anglophones quant aux occasions de maintenir et d'améliorer leurs habiletés en français?

Par ailleurs, comment les universités doivent-elles répondre aux besoins de ces étudiants? Quelles possibilités ces institutions offrent-elles actuellement à cette clientèle? Devrait-on changer les programmes universitaires actuels pour elle? En résumé, quelles sont les implications de l'immersion pour ces étudiants et pour les universités canadiennes?

\section{LA RECHERCHE}

Cet article traitera de ces questions en présentant certains des résultats obtenus dans une recherche entreprise en 1985 par notre groupe de chercheurs de l'Institut des langues secondes de l'Université d'Ottawa et du Centre de Recherche du Conseil Scolaire d'Ottawa. ${ }^{2}$

Notre étude a débuté par une enquête auprès des universités les plus fréquentées par les diplômés de l'immersion de la région Ottawa-Carleton. Dans un deuxième volet, nous nous sommes penchés plus précisément sur deux groupes d'étudiants, soit les anglophones ayant diverses expériences en français qui sont entrés à l'Université d'Ottawa en septembre 1987, et un groupe de diplômés de 1985 des programmes bilingues de la région Ottawa-Carleton. Ainsi, en plus de recueillir des renseignements sur les anglophones faisant leur entrée à l'Université d'Ottawa, cette étude examine en détail les caractéristiques d'un groupe de diplômés des programmes bilingues, à savoir leurs habiletés en français, leur emploi actuel et projeté du français, leurs attitudes langagières et leurs ambitions quant à leurs études universitaires et à leurs carrières éventuelles. Nous sommes actuellement en train de revoir ce groupe alors que les étudiants en sont à leur troisième année d'études universitaires. Regardons maintenant les résultats obtenus jusqu'ici afin de formuler quelques idées concernant les implications des programmes d'immersion pour les départements de français dans les universités.

\section{LES RÉPONSES DES UNIVERSITÉS AUX DIPLÔMÉS D'IMMERSION}

Quelles ont été jusqu'à présent les réponses des institutions aux anciens étudiants d'immersion qui arrivent de plus en plus nombreux au niveau universitaire? Les quatre universités les plus souvent choisies par les diplômés des programmes bilingues au niveau secondaire dans la région d'Ottawa, soit les universités d'Ottawa, Carleton, Queen's et McGill, ont été étudiées du point de vue de ce qu'elles offrent actuellement aux étudiants avancés en français. Déjà en 1985, des administrateurs, dans chacune de ces institutions, envisageaient le développement de stratégies à long terme pour répondre aux besoins de ces étudiants.

De toute évidence, les cours de français et les cours donnés en français représentent pour ces étudiants le moyen le plus important de maintenir et d'améliorer leurs habiletés dans cette langue. Dans l'ensemble, les cours offerts 
aux étudiants en question comptent divers cours avancés de langue seconde, des cours de langue et de littérature françaises, et des cours "encadrés", c'est-à-dire donnés en français dans certaines disciplines et jumelés à des cours spécialisés de français langue seconde. Les cours de traduction, de linguistique, de didactique des langues ainsi que l'apprentissage d'autres langues, l'espagnol par exemple, présentent également pour ces étudiants un intérêt tout spécial.

En plus des cours proprement dits, ces institutions ont pris différents moyens pour encourager l'utilisation du français chez leur clientèle anglophone. Parmi ces moyens on retrouve:

- de la publicité pour les programmes et services disponibles en français

- un coordonnateur chargé de formuler et de mettre en oeuvre la nouvelle politique linguistique

- des exigences en français langue seconde

- un diplôme reconnaissant la compétence en langue seconde

- des programmes de premier cycle (donnés en anglais) qui comprennent l'étude du français

- des services et activités para-scolaires (le Club francophone, le Centre français, la Table française, les étages français dans les résidences).

On retrouvera des renseignements détaillés sur les programmes des universités canadiennes dans les brochures publiées par l'association "Canadian Parents for French" sous le titre: French: It makes a difference. ${ }^{3}$

\section{LES ÉTUDIANTS ANGLOPHONES ADMIS À L'UNIVERSITÉ D'OTTAWA (1987)}

Nous avons élaboré, avec le Bureau de Recherche Institutionnelle, un questionnaire que l'université a fait parvenir aux étudiants anglophones admis à l'Université d'Ottawa. Ce questionnaire s'adressait tout particulièrement aux étudiants possédant une certaine expérience d'une formule d'enseignement "immersif" (programmes réguliers d'immersion, enseignement des différentes matières en français, écoles francophones et cours intensifs d'été). Notre but était de recueillir des informations concernant les programmes de français suivis par ces étudiants au secondaire, et leurs intentions quant à la poursuite de leurs études universitaires dans cette langue.

Les résultats obtenus auprès de la population étudiante anglophone et allophone faisant son entrée à l'université en septembre 1987 nous indiquent que quelque $20 \%$ de ces étudiants ont suivi un programme régulier d'immersion précoce ou tardive (plus ou moins répartis de façon égale dans ces deux programmes), que 13\% sont diplômés d'un programme bilingue au secondaire (la majorité d'entre eux ayant préalablement complété un programme d'immersion précoce ou tardive) et que quelque $5 \%$ de ces étudiants proviennent des écoles françaises pour francophones. Un autre $5 \%$ ont reçu, en plus du programme cadre de français, l'enseignement en français d'une matière au niveau secondaire, et enfin $8 \%$ déclarent avoir suivi d'autres programmes spéciaux de français (des échanges, des 
programmes intensifs d'été, etc.). Lorsqu'on songe qu'il y a quinze ans ces possibilités existaient à peine, ces résultats sont, en vérité, remarquables.

Deux tiers des étudiants qui ont eu une certaine expérience "immersive" avant de venir chez nous ont indiqué qu'ils avaient l'intention de poursuivre leurs études en français à l'université. Cette tendance était plus apparente parmi les étudiants ayant fait des études bilingues au secondaire, avec $76 \%$ de réponses positives. Trente-cinq pour cent (35\%) de tous les répondants avaient l'intention de prendre un cours ou deux en français, $23 \%$ de suivre "quelques" cours en français, et $8 \%$ de suivre la "majorité" de leurs cours en français. Les principales raisons évoquées étaient le désir de devenir bilingue et la nécessité de parler français couramment pour faire carrière. Par ailleurs, les $34 \%$ de répondants n'ayant pas l'intention d'entreprendre des études en français ont expliqué que s'ils faisaient leurs études en français leurs notes risqueraient d'en souffrir, et que, de toute façon, ils pensaient ne pas avoir assez de temps, étant donné les exigences de leurs programmes.

Les constatations découlant de toutes ces données peuvent servir de point de départ à l'élaboration de suggestions qui permettraient d'encourager cette clientèle étudiante. Les institutions pourraient, par exemple, offrir des cours d'intérêt personnel (littérature, conversation, culture) et de préparation aux carrières (rédaction administrative, le français spécialisé des sciences de la santé ou encore le français diplomatique ou des affaires). Les données préconisent également des cours offerts en français dans diverses matières universitaires telles que la biologie ou la géographie, cours qui seraient intégrés aux programmes menant à un diplôme. Des options pourraient être proposées aux étudiants craignant de voir baisser leur moyenne: remplacer les notes par la formule "réussite-échec", ajuster les tâches de façon à souligner les habiletés réceptives, ou encore, présenter des critères d'attribution de notes accordant plus d'importance à la maîtrise du contenu du cours qu'à celle de la langue.

\section{LES DIPLÔMÉS DES PROGRAMMES BILINGUES DU SECONDAIRE DE 1985}

Le reste de cet article sera consacré à notre étude du groupe de diplômés de 1985 des programmes bilingues au niveau secondaire. En juin 1985, quelque 300 étudiants ont obtenu leurs diplômes des programmes bilingues des Conseils scolaires d'Ottawa et de Carleton. Tous avaient obtenu 10 à 13 crédits en français au niveau secondaire, c'est-à-dire un tiers de leurs cours. Environ $40 \%$ de ces étudiants étaient du groupe "Jardin d'enfants de 1971" et avaient débuté 14 ans auparavant dans un programme d'immersion précoce. Cette séquence d'immersion précoce suivie de l'école secondaire bilingue représente 6500 à 7000 heures d'enseignement scolaire en français. D'autres étudiants se sont joints au groupe en sixième ou septième année en entrant dans les programmes d'immersion tardive, ce qui représente 3000 à 4000 heures d'enseignement en français. 
Des études antérieures (McGillvray et Pawley, 1985; Morrison et Pawley, 1986) avaient démontré que la grande majorité (presque $90 \%$ ) de ces étudiants avaient l'intention d'aller à l'université après leur $13^{\mathrm{e}}$ année, et qu'ils s'orientaient vers une grande variété de champs d'étude. Il n'y avait aucune indication que ceux-ci entreraient plus que d'autres dans les programmes de langue et de littérature françaises; au contraire ils semblaient s'orienter davantage vers les sciences et la technologie que vers les langues et les humanités.

\section{LES SUJETS}

Parmi les diplômés de 1985, nous avons pu identifier 81 étudiants faisant leur entrée dans les quatre universités en question cet automne-là et nous leur avons administré les questionnaires et les tests. Le sous-groupe le plus nombreux, qui se compose de 34 étudiants, s'est dirigé vers l'Université d'Ottawa. Carleton en a reçu 28, Queen's 14 et McGill 5 (voir tableau I).

En 1988, nous avons réussi à reprendre contact avec la majorité des étudiants du groupe original et avons administré les mêmes instruments de mesure à 48 des étudiants actuellement inscrits aux universités d'Ottawa, Carleton et Queen's.

Tableau 1

DIPLÔMÉS DES PROGRAMMES BILINGUES DES CONSEILS SCOLAIRES D'OTTAWA ET DE CARLETON PARTICIPANT À L'ÉTUdE

\begin{tabular}{lcccc}
\hline & $\begin{array}{c}\text { Sujets évalués } \\
\text { en } 1985\end{array}$ & \multicolumn{2}{c}{$\begin{array}{c}\text { Sujets réévalués } \\
\text { en } 1988\end{array}$} \\
\hline Université d'Ottawa & 34 & & 24 & \\
Carleton & 28 & & 16 & \\
Queen's & 14 & & 8 & \\
McGill & 5 & & 48 & (19 précoce, \\
\hline & 81 & (33 précoce, & 49 tardive) \\
\hline
\end{tabular}

\section{LES INSTRUMENTS}

En 1985, pendant leur première année d'études universitaires, tous les étudiants ont subi un test de compétence en français (compréhension, lecture, vocabulaire, composition, expression orale $)^{4}$ et rempli un questionnaire d'auto-évaluation de leurs habiletés réceptives en français dans des situations quotidiennes (compréhension auditive et lecture). Ils ont complété un autre questionnaire détaillant leurs habitudes de l'utilisation du français et leurs attitudes à l'égard de cette utilisation (leur degré d'assurance à parler français, les raisons les poussant à apprendre le français, etc.). Ils ont également participé à une entrevue individuelle en français langue seconde afin que l'on puisse évaluer leurs habiletés en expression orale. 


\section{LES RÉSULTATS}

\section{Compétence en français}

Les résultats obtenus au test écrit en 1985 démontraient chez ces étudiants un niveau de compétence très élevé en compréhension orale, en lecture et en composition. Les résultats obtenus à l'entrevue leur attribuaient également un niveau assez élevé d'habiletés en expression orale (Wesche, Morrison, Pawley et Ready, 1986). Quoique les résultats obtenus à ces deux tests soient en-dessous des moyennes obtenus par les locuteurs natifs, il semble que les diplômés des programmes bilingues ont entrepris leur première année d'études universitaires avec un degré de compétence à communiquer en français relativement élevé qui devait leur permettre, dans la majorité des cas, de suivre des cours s'adressant à des francophones.

Les réponses recueillies en 1985 (voir Leblanc et Painchaud, 1985) par le questionnaire d'auto-évaluation des habiletés réceptives semblent indiquer que ces étudiants démontraient une grande confiance quant à leur capacité de comprendre et de lire le français dans les situations quotidiennes telles que:

“Au téléphone, lorsqu'une personne ne parle qu'en français, je peux comprendre ce que demande cette personne"

et

"Si j'emprunte à un ami ses notes de cours écrites en français je peux m'en servir pour mon devoir du jour"

D'autres données indiquent toutefois qu'ils étaient moins sûrs d'eux au niveau de la production (i.e. l'expression orale et écrite) qu'au niveau réceptif.

Les différences rencontrées entre les diplômés de l'immersion précoce et ceux de l'immersion tardive étaient minimes. Bien que les étudiants de l'immersion précoce aient été quelque peu favorisés au niveau de l'expression orale et de la confiance en soi, les résultats ne présentent pas de différence significative lorsque les habiletés réceptives sont prises en considération. En fait, la différence réelle entre les deux groupes n'est pas très grande, et ce, particulièrement quand on considère les habiletés reliées à l'environnement académique. Les diplômés des programmes d'immersion précoce et tardive représentent des populations quelque peu différentes et il n'est évidemment pas possible d'interpréter ces données de façon à comparer l'efficacité des deux programmes. ${ }^{5}$

\section{Attitudes à l'égard du français}

Les réponses au questionnaire analysant les attitudes par rapport à la langue seconde ont démontré un ferme désir, de la part des étudiants de la région d'Ottawa, de se servir du français et ont révélé de multiples raisons pour apprendre à le parler. Toutes les raisons énumérées dans le questionnaire ont été jugées 
appropriées par les étudiants: de meilleures possibilités d'emploi, de meilleures occasions de voyager et de rencontrer des francophones de même que l'intérêt et la satisfaction de pouvoir parler une langue seconde. Ils croyaient aussi que le fait de pouvoir s'exprimer dans les deux langues officielles pourrait contribuer à renforcer l'unité nationale.

\section{L'emploi du français}

Les réponses de ces diplômés des programmes bilingues au questionnaire administré en 1985 concernant leur emploi du français en première année d'études universitaires nous donne l'image suivante:

1 Fréquence de l'emploi du francais. La moyenne de l'usage du français telle qu'estimée par les étudiants eux-mêmes n'était pas très élevée, suggérant l'emploi occasionnel de la langue dans une variété de situations. Quoique plus élevée pour ceux de l'immersion précoce que pour ceux de l'immersion tardive, l'écart entre les deux groupes demeurait toutefois minime.

2 Changements dans l'usage du français. En général, tous les groupes, excepté celui de l'Université d'Ottawa, ont rapporté qu'en moyenne ils se servaient moins du français en première année d'université que lorsqu'ils étaient en treizième au secondaire. Avec le groupe de l'Université d'Ottawa il n'y avait cependant pas, à ce sujet, de changements à signaler. Ces conclusions sont d'autant plus frappantes qu'un bon nombre de ces étudiants terminent leur programme bilingue en douzième année et que la plupart d'entre eux ne suivent qu'un cours de français en treizième année. D'autre part, tous les groupes prévoyaient que leur emploi du français irait en augmentant à l'avenir par rapport au présent.

3 Emplois spécifiques du français. Des mesures spécifiques de l'usage courant du français indiquent encore une fois que la majorité de ces étudiants emploient relativement peu le français hors de la salle de classe. Les données obtenues sont les suivantes: les étudiants de la région d'Ottawa suivent de zéro à trois cours en français (incluant les cours de langue) pendant leur première année universitaire, soit une moyenne d'un demi cours; ils regardent environ deux heures de télévision en français par mois, lisent un livre en français tous les trois mois, regardent un film en français tous les deux mois et se servent du français à peu près quatre heures et demie par mois dans d'autres occasions.

\section{Réadministration des instruments en 1988}

Au printemps 1988, nous avons réussi à réadministrer les tests et le questionnaire à ces mêmes étudiants de la région d'Ottawa. Si l'on compare les résultats obtenus aux sous-tests qui sont restés les mêmes et si l'on considère seulement les scores des 48 étudiants qui ont passé ces tests en 1985 et en 1988, on peut dire que dans 
l'ensemble ces étudiants ont maintenu leurs habiletés au niveau de l'expression orale et ont démontré une amélioration significative au niveau de la compréhension auditive (voir tableau II) ${ }^{6}$. Pour ce qui est de la lecture et de la compréhension auditive, ils ont obtenu des résultats correspondant aux normes "locuteur natif" établies pour des universitaires francophones. On a cependant noté une baisse dans l'ensemble des notes au test de closure, test pour lequel il y avait, comme pour la dictée, des différences significatives entre les diplômés de l'immersion et les locuteurs natifs. Quant aux attitudes envers l'emploi du français, le seul changement que l'on ait remarqué par rapport à la première année était une augmentation du taux d'anxiété, probablement reliée à la baisse légère, mais significative, de l'utilisation du français. Mentionnons que les étudiants de l'Université d'Ottawa démontraient certains avantages vis-à-vis des autres, notamment au niveau du nombre de cours suivis en français pendant leurs trois années universitaires.

\section{IMPLICATIONS}

Quel type de cours en français attire les diplômés de l'immersion, et quelles conclusions pratiques peut-on tirer de tout cela pour les programmes français de nos universités?

Dans notre institution, l'expérience a démontré que ces diplômés sont de bons candidats pour toute une gamme de cours offerts en français. L'Université d'Ottawa étant bilingue, ces cours incluent aussi bien ceux qui sont destinés aux anglophones qu'aux francophones. (C'est aussi le cas pour les cours de langue et de littérature françaises. L'Institut des langues secondes offre des cours de français langue seconde et le Département de Lettres françaises offre des programmes pour les francophones et pour les anglophones très avancés et français). Nos étudiants anglophones qui atteignent un niveau élevé de compétence en français ont l'occasion de suivre des cours destinés aux francophones dans toutes les matières. Ceci devrait, à mon avis, être le but ultime des diplômés vraiment intéressés. Ailleurs, ce but devrait être réalisé grâce à des échanges inter-universitaires.

On retrouve ces même étudiants dans les cours de langue et littérature françaises ainsi que ceux de civilistion canadienne et française au Département de Lettres françaises, de même que dans plusieurs autres disciplines. Mentionnons qu'ils suivent également des cours avancés en français langue seconde à l'Institut des langues secondes. On a déterminé le nombre de cours suivis en français par un groupe de diplômés de l'immersion de 1985 qui sont restés à l'Université d'Ottawa. Pendant leurs trois années d'études, plus de $80 \%$ de ces étudiants ont suivi des cours en français. Ces derniers en ont suivi une moyenne de 4 par étudiant, dont $28 \%$ sont des cours de français langue seconde destinés aux anglophones, et $72 \%$ sont des cours destinés aux francophones (12\% en Lettres françaises et $60 \%$ dans des disciplines variées). Ainsi, même dans notre université, la plupart de ces étudiants choisissent de prendre la majorité de leurs cours en anglais. Le fait d'avoir la possibilité de suivre des cours en français 
Tobleou II

Diplômés de l'immersion et atudionts universitaires francophones. Moyenne des notes ot différences de groupo obtenues aux mesures d'évaluation de la compétence langogióre

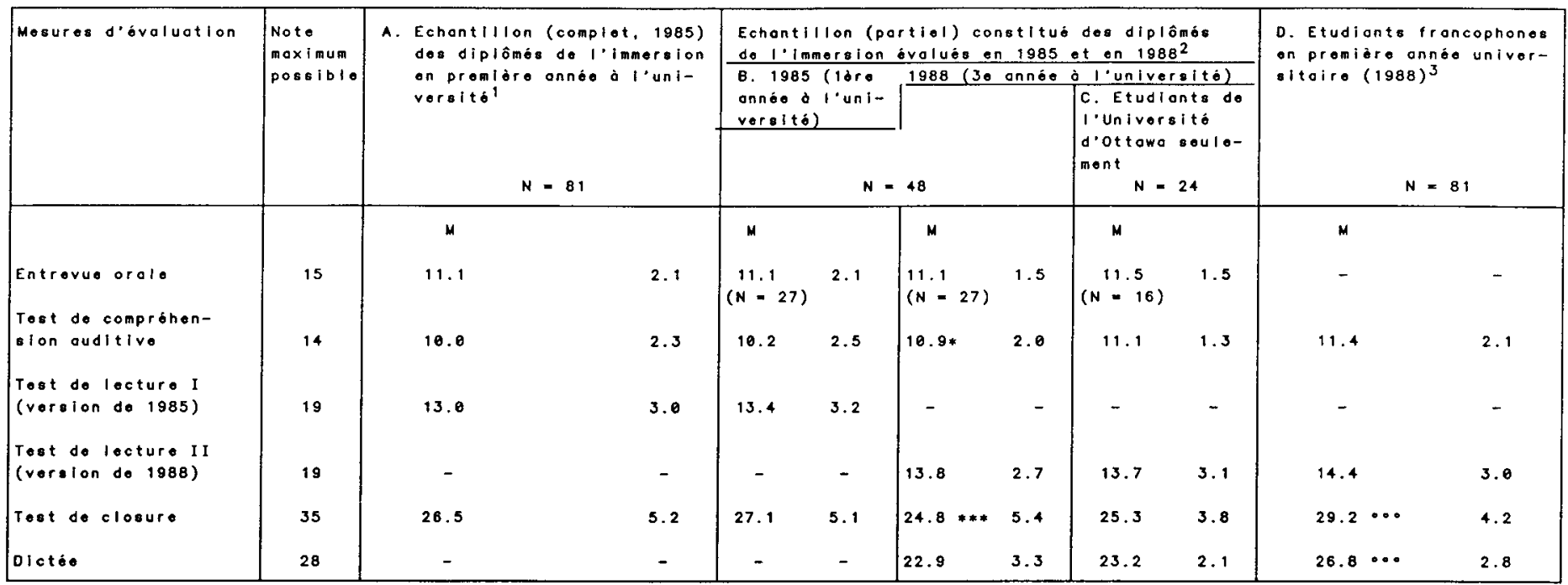

1. On n'o trouvé oucune différence signiflcotive entre le groupe $A$ ot 10 soue-groupe $\theta$.

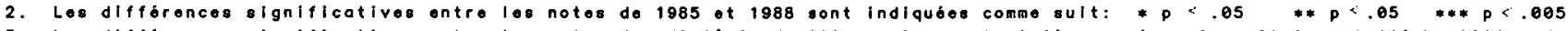

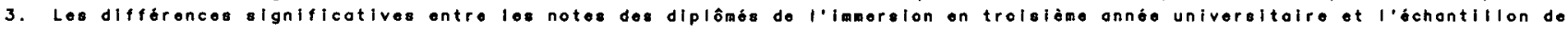
francophones sont indiquáos comme ult: $p<.05$. $p<.01 \quad \cdots p<.005$ 
destinés aux francophones n'assure donc pas que les diplômés d'immersion sont toujours prêts à faire face au défi que représentent de tels cours. Même s'ils en sont capables, ils ne veulent souvent pas prendre un tel risque. Ils en craignent trop les conséquences, à savoir les mauvaises notes dues à la concurrence avec des étudiants francophones. Le risque est naturellement plus grand avec les cours qui exigent beaucoup de travail écrit et des présentations orales ou des discussions.

A part les cours actuellement offerts en littérature, en civilisation et en stylistique (qui, dans notre univerșité, sont principalement destinés aux étudiants francophones), que peuvent offrir les départements de français pour encourager ces étudiants à poursuivre leurs études en français?

Des cours de langue seconde. A l'Institut des langues secondes, on remarque que les cours de FLS correctifs axés sur leurs points faibles (cours avancés de composition, d'expression orale et de grammaire) sont particulièrement efficaces. On retrouve ces étudiants dans notre centre d'auto-apprentissage où ils travaillent de façon autonome, soit par la lecture de livres et de revues, soit par le visionnement de vidéo-cassettes (films, émissions de télévision), soit par l'utilisation de l'ordinateur. Un professeur de langue est disponible afin d'orienter et d'aider les étudiants.

Des cours dans les matières donnés en français. Nous avons également tenté d'encourager nos étudiants à faire une partie de leurs cours en français dans d'autres disciplines, et ce, de diverses manières. Notre situation unique nous permet d'offrir deux types de cours encadrés axés sur la matière. Entre 1981 et 1986, on se servait de l'ancienne formule, déjà en vigueur dans d'autres universités, sorte d'immersion tardive au niveau universitaire où, par exemple en psychologie, une section a été réservée pour les locuteurs non natifs (Burger, Chrétien, Gingras, Hauptman et Migneron, 1984; Edwards, Wesche, Krashen, Clément et Kruidenier, 1984; Wesche, 1985). Le professeur de psychologie est alors secondé dans ses classes par un professeur de FLS. Ces cours sont de toute évidence excellents pour les étudiants sortant des meilleurs programmes cadres de français. Selon moi, la majorité de ceux qui proviennent des programmes bilingues au niveau secondaire ne pourraient cependant pas tirer grand avantage de cette situation protégée (qui représenterait une continuation de leur expérience en français au niveau secondaire). Ces cours n'amélioreraient pas tellement leurs habitudes langagières car ils sont conçus dans le but de répondre aux demandes langagières réduites et donc ajustées au niveau des locuteurs non natifs.

Sous la pression des contraintes budgétaires on expérimente depuis 1984 une autre formule "encadrée", formule qui donne des résultats dépassant même nos attentes (Migneron et Burger, 1986). Ce sont des cours avancés de langue jumelés à des cours populaires dans ces disciplines variées (pour le moment psychologie, sociologie, histoire, linguistique et sciences politiques). Les cours de langue, crédités, sont spécialement conçus pour fournir aux étudiants le matériel linguistique nécessaire à une bonne assimilation de la matière académique présentée dans l'autre cours. Cette nouvelle formule semble offrir à nos étudiants une solide préparation et leur servir de transition vers les cours non encadrés 
destinés aux francophones. Nos professeurs de langue aiment enseigner ces cours de langue jumelés, même si cela implique un certain travail supplémentaire, parce qu'ils peuvent constater le progrès et la satisfaction des étudiants.

\section{CONCLUSION}

En résumé, on pourrait affirmer que des anglophones fonctionnellement bilingues commencent à arriver dans nos universités, qu'ils déclarent vouloir maintenir et améliorer leurs habiletés en français, et qu'il y a parmi eux des étudiants qui saisissent chaque occasion pour le faire. Cependant, il est aussi évident que le français n'est pas une priorité pour la majorité d'entre eux, et que si les départements de français désirent les attirer, il faudra, sans se permettre de délaisser le riche contenu traditionnel en littérature et en culture, leur offrir aussi des cours qui répondent d'une façon précise à leurs besoins, c'est-à-dire à leurs faiblesses en langue seconde, à leurs craintes du risque et à leurs intérêts personnels, professionnels et académiques.

\section{NOTES}

1 Cet article a déjà paru dans C. Besnard et C. Elkabas (Eds.) (1988). L'université de demain: courants actuels et apports de la didactique des langues à l'enseignement du français langue seconde. Toronto: Canadian Scholars' Press Inc.

2 L'étude pilote de 1985 a été subventionnée par le Programme des langues officielles en éducation du Secrétariat d'Etat. Le Ministère des collèges et universités de l'Ontario subventionne actuellement la poursuite de cette étude. Nombreux sont ceux qui nous ont aidés à faire cette recherche. Je voudrais mentionner en particulier les membres de notre équipe: Mme Doreen Ready, le Dr Frances Morrison, Mme Catherine Pawley, Mme Jacqueline Murray, Mme Deborah Lawson, Mlle Sarah Thomas ainsi que le Dr.Christiane Hamm qui a collaboré avec nous à Queen's. Je voudrais remercier également $M$. Yves Benac et $M$. Gerry Martineau du Bureau de la Recherche institutionnelle de l'Université d'Ottawa de même que Mme Laure Krupka et Mme Michèle Thibodeau pour leur appui dans la rédaction de ce texte français.

3 On peut se procurer cette documentation en écrivant à l'adresse suivante: Canadian Parents for French, 309 rue Cooper, Suite 210, Ottawa (Ontario) K2P 0G5.

4 Nos instruments sont disponibles sous une forme révisée pour les chercheurs désirant étudier des populations semblables. Voir Wesche, Morrison, Pawley et Ready, 1986 pour plus de détails.

5 L'étude actuelle, portant sur le deuxième groupe de diplômés de l'immersion (1988), ne démontre pas de différences entre les anciens étudiants d'immersion précoce et ceux d'immersion tardive.

6 Les résultats obtenus avec ces 48 étudiants ne présentaient aucune différence significative avec ceux de l'ensemble du groupe de 81 étudiants, et ce pour chacun des instruments d'évaluation utilisés.

\section{REFERENCES}

Burger, S., Chrétien, M., Gingras, M., Hauptman, P., \& Migneron, M. (1984). Le rôle du professeur de langue dans un cours de matière académique en langue seconde. Revue canadienne des langues vivantes, $4 I(2), 397-402$.

Canadian Parents for French. (1987). French: it makes a difference. Ottawa: Canadian Parents for French. 
41 Les diplômés de l'immersion: implications dans le domaine de l'enseignement du français

Edwards, H., Wesche, M., Krashen, S., Clément, R., \& Kruidenier, B. (1984). Second language acquisition through subject-matter learning: a study of sheltered psychology classes at the University of Ottawa. Revue canadienne des langues vivantes, 41(2), 268-282.

Leblanc, R., \& Painchaud, G. (1985). Self-assessment as a second language placement instrument. TESOL Quarterly, 19, 673-687.

McGillvray, W. R., \& Pawley, C. (1985). Grade 13 student survey 1985. Unpublished questionnaire, Ottawa Board of Education.

Migneron, M., \& Burger, S. (1986). Teaching strategies for immersion courses. Canadian Association of Immersion Teachers, 9(1): 10-11.

Morrison, F., and Pawley, C. (1986). French proficiency of immersion students at the Grade 12 level. Toronto: Ontario Ministry of Education.

Wesche, M., Morrison, F., Pawley, C., \& Ready, D. 1986. Post-secondary follow-up of former French immersion students in the Ottawa area. Report to the Language Acquisition Development Program, Department of the Secretary of State.

Wesche, M., Morrison, F., Ready, D., \& Pawley, C. 1990. French immersion: post-secondary consequences for individuals and universities. Revue canadienne des langues vivantes, $46(3), 1-21$. 